\title{
Hit2Gap Project: Highly Innovative building control Tools Tackling the energy performance gap
}

\author{
Andrea Costa $^{1, *}$, Marco Pietrobon ${ }^{1}$, and Thomas Messervey ${ }^{1}$. \\ ${ }^{1}$ R2M Solution Srl - Research to Market Solution, 27100 Pavia, Italy
}

\begin{abstract}
Measurement campaigns have shown major discrepancies in buildings energy performance between planned energy demand and real energy consumption, while nowadays most of the newly constructed offices buildings are equipped with BMS systems, integrating a more or less extended measurement layer providing large amounts of data. The HIT2GAP project has developed a new generation of building monitoring and control tools based on advanced data treatment techniques allowing new approaches to assess building energy performance data, getting a better understanding of building's behaviour and hence a better performance. From a strong research layer on data, HIT2GAP solution builds on existing measurement and control tools that are embedded into a new software platform for performance optimization. The HIT2GAP solution is applied as a novel intelligent layer offering new capability of the existing BMS systems and offering the management stakeholders opportunities for services with a novel added value. Applying the solutions to groups of buildings also allows to test energy demand vs. local production management modules. This solution is being tested in various pilot sites across Europe. HIT2GAP work has been carried out with a permanent concern about market exploitation of the solutions developed within the project. This paper will present the project solution in detail and showcase the achievement so far in the real case demo sites.
\end{abstract}

\section{Introduction}

Differences between expected and actual energy consumptions in buildings arise from issues arising from the design, construction, commissioning and operation. This Energy Performance GAP, which often exceeds $50 \%$ of the overall energy consumption, has detrimental implications for the achievement of EU energy targets.

The objective of the HIT2GAP project is (i) to reduce the energy performance gap between predicted and actual building performance, focusing on the operational phase of buildings; (ii) to propose a new paradigm for the development of energy management platforms in buildings, integrating existing expertise and resources; (iii) to provide a smart, open platform associated with added-value modules based on analytics of data collected in the building.

Funded by the European Union under the Horizon 2020 research and innovation programme, HIT2GAP is active from September 2015 to August 2019.

Demonstration of the developed solution in four pilot sites and evaluation of the impact and savings achieved using this solution.

\section{Project solution}

The main objective of HIT2GAP is to reduce the gap between planned energy demand and real energy consumption in buildings.
The approach taken in HIT2GAP is to build an open, plug and play, application-based platform to support a new generation of Building Management Systems (BMS). This approach is seen as the effective strategy to cover user requirements in buildings that by their nature (typology, climate, usage) are widely diverse. Furthermore, it allows the platform to evolve and remain up-to-date with new emerging technologies in order to better assess energy use within a building or a block of buildings. Thus, by design the HIT2GAP platform is required to be modular and interoperable [1].

Although the approach proposed by HIT2GAP is generic, the project focusses on the gap reduction in commercial buildings during the commissioning and operation phases of the buildings life cycles. However the results generated by the HIT2GAP project in terms of modelling of the building behaviour may be of value to the design and construction phases of future buildings.

The HIT2GAP methodology aims to build a new generation of building monitoring and control tools based on advanced data treatment techniques allowing new approaches to assess the energy performance of buildings, getting a better understanding of the building's behaviour and hence a better performance.

HIT2GAP will enhance current BMS functionalities by:

- Using alternative sensors and meters (i.e., smart phones, mobile phones, laptops, tablets, smart watches, etc.) to capture building complexities by monitoring

\footnotetext{
* Corresponding author: andrea.costa@r2msolution.com
} 
spaces lacking traditional sensors or to extract additional information for existing data sources (i.e. disaggregation).

- Integrating Data Mining techniques for Knowledge Discovery (DMKD) as a core module for building performance assessment and understanding, and associated predictive maintenance services.

- Adding occupants' behaviour to the equation. Taking into account that users also play a significant role in building performance, it will be possible to better understand building performance, enhance predictions and control and optimise energy consumption and occupants' comfort/health.

- Providing continuous performance evaluation and improvement based on measured, historical and simulated data coming from a new generation of simulation services.

- Integrating demand/supply management tools for building-integrated and community renewable energy sources.

- Adapting the visualization interfaces to specific user needs (technical and non-technical profiles) to deliver the information effectively.

\subsection{Core platform}

The HIT2GAP solution is represented in the scheme in Fig. 1. It is based on an open platform which allows the connection and integration with several and extendible informatic modules with different functionalities (application level in Fig. 1). The core platform provides also basis functionalities such as data structuring (data model based on ontologies covering the whole building), data pre-processing (consolidation and alerts, conversion, arithmetic functions, detection of outliers and missing data, extrapolation), data storage.
The data storage management is based on specific applications integrated in the project such as MONDAS (Monitoring and Data Analysis System: a platform for the acquisition, the management, the real-time analysis, developed at the Fraunhofer Institute for Solar Energy Systems ISE over the last 10 years), HDF5 (Hierarchical Data format), and JENA (an informatic project, built on the Java programming language).

The Hit2Gap Core Platform is a central element that collect information from a wide-range of sources (files, sensors, BMS); the collected data are diverse in their format, in their nature (static data such as descriptive data of the building vs. dynamic data coming from sensors, or wearable devices). It is also able to provide the information required by data analysis or visualization modules. The platform is also to collect the information generated by third-party modules so as to make it available to other third-party modules

In order to achieve these data transfer functionality, the Core Platform needs to structure the data that comes from multiple sources. A data model will be developed so as to exchange and share data in a smarter and standardized way, homogenising the existing building protocols under a common vocabulary.

Additionally, to these two fundamental functionalities, some functionalities would widely be appreciated by third-party modules: - to provide quality with the data: data can be altered because of different reasons and third-party modules should be able to identify data that are altered, and if possible, to be provided with a corrected data; - to provide analytics solution to the third-party modules so that a core platform client can pre-process the data that will become the input of its module.

Finally, in order to make such functionality works, some user interface has been developed and integrated.

The platform itself offers also visualisation

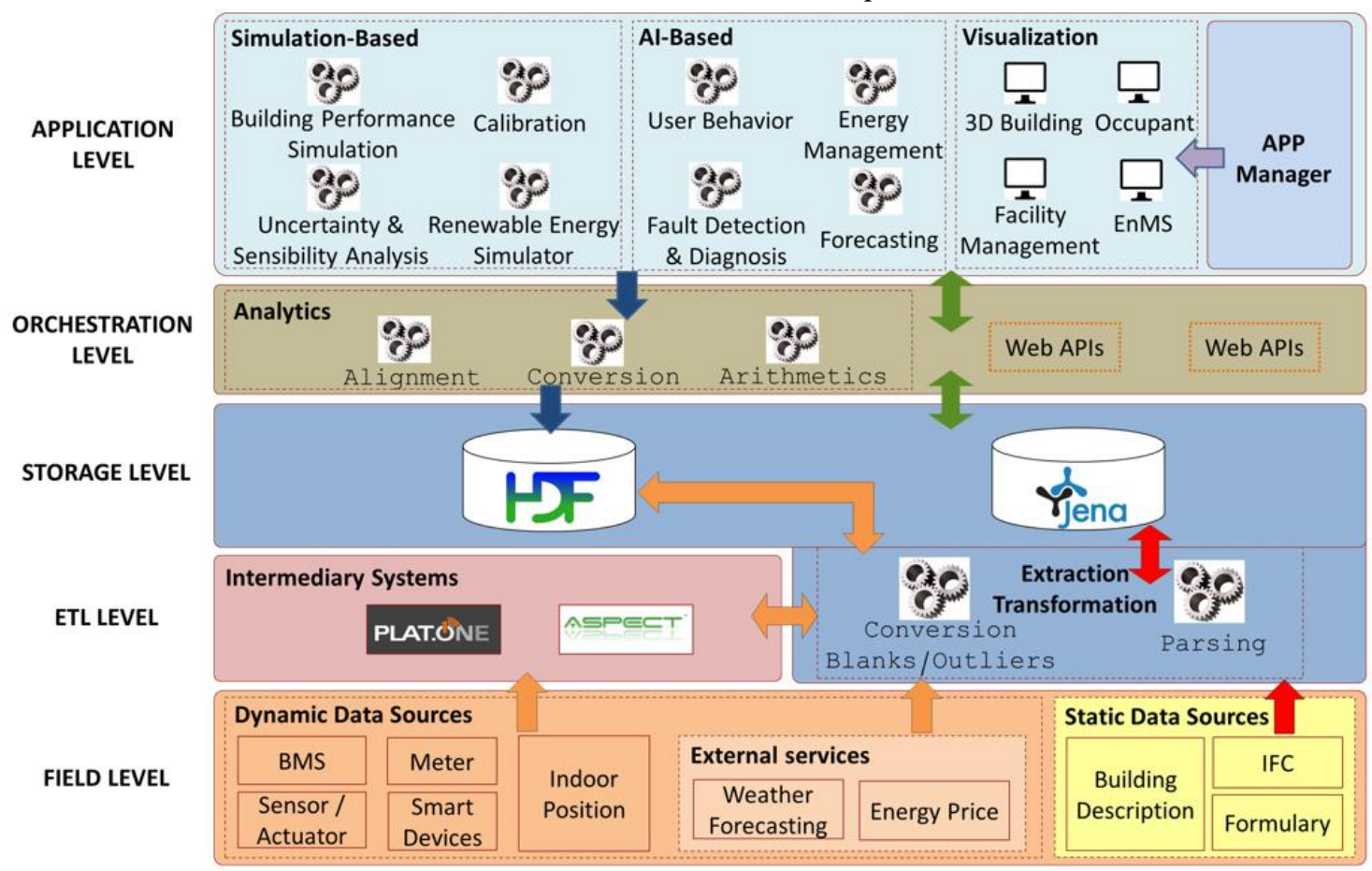

Fig. 1. Overall architecture of the Hit2Gap solution: open, modular and evolutive data platform. 
functionalities of data trends and of results from the linked modules.

\subsection{Data collection}

In order to provide data to the platform and to collect them from the field level (Fig. 1), two different approaches are possible and described here. The first one is the usage of a middleware platform that provides a wide set of services like data connector, data persistency, real time data consumption and a wide set of API that guarantee the necessary access points for the application to access and use the collected data. This approach has been achieved by using the IoT Enterprise Grade platform called Plat.One and developed on its base the necessary data connector to each building. It enables the users of HIT2GAP to transfer data from their system to the platform, whether they are using existing or new protocols. The other possible approach is the direct connection from a BMS to HIT2GAP data model layer. Indeed the platform provides all necessary tools to integrate directly a BMS system or other data sources to the platform avoiding any intermediate level.

Data from Plat.One or directly from BMS communication tools are sent at a configurable frequency by calling the Core Platform API.

\subsection{Modules}

Nine different modules plus the specific display ones, all described hereafter, have been developed by the project consortium. Some are related to same thematic area but with different features. The core platform and some modules will be available for free, at the end of the project. The core platform allows for integration of possible further modules in the future [2]

\subsubsection{Energy management}

The Energy Management (EM) module has the capability of supporting all aspects of an Energy Management System (EnMS) in any building or building stock belonging to Organisations of any type and size. The EM module is an adaptation of the Enerit commercial cloud-based ISO 50001 [3] software which, unlike energy monitoring and energy data reporting, guarantees a systematic approach to Energy Management in compliance with the ISO 50001 international standard, such as plans, actions, communication and documents needed for the standard, in a workflow-based solution.

\subsubsection{Load Forecasting}

Load Forecasting module (example of interface view in Fig. 2), based on historical data, offers functionality is useful to support different energy management activities as energy budget control (monthly forecasting), demand response (short-term forecasting, such as hourly predictions) and performance measure and verification protocols.

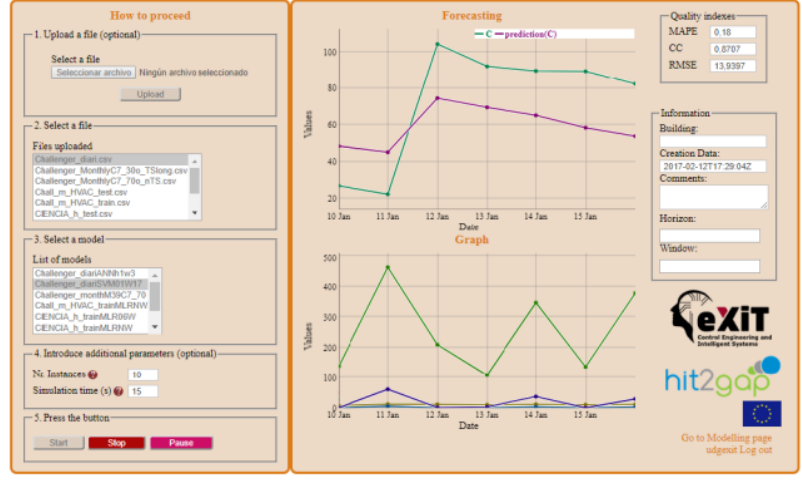

Fig. 2. Example of Load forecasting results visualisation.

\subsubsection{Fault Detection and diagnosis}

Fault detection and diagnosis (FDD) modules enable the early detection of system and component malfunctions that deteriorate the energy performance of buildings and provide information facilitating the application of corrective actions.

Two FDD algorithms will be used in HIT2GAP: a method based on Decision Trees (ML) and a method based on a Density Based Clustering Algorithm (DBSCAN, Principal component analysis - PCA).

HIT2GAP has developed a facility for fault detection and diagnosis using principal component analysis. This works by comparing current data against a reference model which has been constructed from historical data. The module behind this service, known as the 'FDDPCA module', analyses the values and variances of the incoming data from a building's sensors, looking for any instances where the incoming data does not fit the model. If an abnormal set of readings is identified, the contribution of each variable is analysed in order to determine which variables were connected with the faulty reading, enabling the nature of the abnormality to be identified.

The Fault Detection and Diagnosis - Principal Component Analysis module (FDD-PCA) is a Data Mining and Knowledge Discovery (DMKD) module that utilises the HIT2GAP integrated data access to offer enhanced monitoring capabilities that evaluate a building's behaviour with respect to normal operation. The analysis is based on a statistical model (FDD model) which is trained beforehand with historical data corresponding to normal operation condition (NOC). This reference model is built on the basis of multivariate statistics theory, known as principal component analysis (PCA). It the user to identify the correlation among all variables selected for monitoring, including their dependencies on the energy consumption, and finds the statistical bounds of such dependencies. This approach allows the definition of two simple monitoring charts showing the independence of the number of variables being monitored, and provides visual information about the building behaviour. Comparison of new observations to the reference model leads to detection of abnormal operating conditions (AOC) as faults and failures in sensors or technical equipment or user misbehaviours. In the case of detecting a fault or an abnormal condition, 
the module allows the user to isolate the variables causing this. Based on this information, maintenance staff can identify causes or explain the reason of the detected fault more easily.

This module is especially intended to support continuous monitoring during the implantation of energy management procedures as ISO 50001 or ISO 50006:2014 (energy performance) and also can be used to support audits (EN16247-1, ISO 50002) and performance measure and verification protocols (IPMVP, ISO 50015:2014).

The second FDD modules developed in the HIT2GAP uses machine learning based (ML) methods developed at Fraunhofer Institute for Solar Energy Systems ISE. These methods continuously analyse timeseries data from the core platform and provide a message to building operators if anomalies are detected. The module is implemented on the MONDAS (MONitoring and Data AnalysiS).

The module allows classification new measurement data of selected HVAC systems as fault-free or faulty after having being trained with labelled data. Additionally, the methods support diagnostic tasks by identifying the sensors responsible for deviations from a nominal (fault-free) operation.

To enable the detection of several classes of faults (e.g. sticking valves, missed set temperatures, schedule mismatch) in different systems (e.g. AHUs, heating circuits), the monitoring data must include predefined minimal data sets and conform with data quality requirements (resolution) Furthermore, training data sets must be provided, i.e. specified during configuration, containing pre-classified faulty and fault-free data for the respective system or system type.

\subsubsection{User Behaviour modelling}

Two modules has been developed and integrated about the user behaviour field, a semantic one and eventdriven one.The aim of the behaviour modules is to deliver user behaviour information to the building users, to highlight energy wastage and to suggest behavioural and systemic changes.

The semantic behaviour module (BM) will provide a better understanding of the drivers of behaviour, with a focus on the users' state or condition. In particular, the BM will take into account building meter readings and the analyses of user behaviour inside the building (movements, occupancy, etc.). This aspect will serve to understand, model and influence users' behaviour with respect to energy consumption indicators. Another key point makes reference to the fulfilment of energy standards and comfort levels. With this aim, the proposed module fully integrates indoor comfort and health issues, especially regarding the following aspects: (i) improving indoor environmental conditions considering thermal preferences and satisfaction of occupants, which can be a positive influence on productivity; (ii) improving ventilation rates to reduce the risk of sick building symptoms (respiratory illness, allergy and asthma symptoms); (iii) reducing occupant- related anomalous energy use and related $\mathrm{CO} 2$ emissions; and (iv) providing continuous monitoring of indoor air quality. The main benefit of this module is to reduce the energy consumption while considering user behaviour and ensuring comfort levels. Moreover, the user behaviour module will inform users about detected inefficiencies in order to involve them in the learning process, to make them aware of issues, and to foster sustainable behaviour strategies in buildings.

The BM is an application module that produces recommendations about the energy wasting states of interactions in the whole system and sends messages to facility managers and other targeted users through the use of semantic reasoning. The BM makes use of the BM ontology which represents all parts of the system (the definitions and instances of the building architecture, the building systems, the working schedules, the occupants, etc.), the relationships between the parts and semantic rules defining energy wasting states which have been discovered by the Knowledge Discovery in Databases (KDD) process.

The event-driven User Behaviour Module (BME) is a typical application providing some distinct functionality, related to the tracking and reporting of the behaviour of the building users. In the BME "behaviour" is considered in terms of events, i.e., specific situations that reveal problematic user behaviour, mainly in terms of energy efficiency. This said, in some cases the event may result in an energy saving practice which is again recorded and credited as such.

In a long term and strategic view of the BME the module will be able to provide for: (i) notifications: to issue notifications to various users as regards the events tracked; (ii) interoperability: to connect and provide data to other modules, applications; such is the case of the Energy Management module, to which it will connect via HIT2GAP; (iii) community gaming: to allow the development of communities via gaming; (iv) control: to support manual or automated, behaviour event- driven, control decisions.

\subsubsection{Renewable Energy Simulator}

Renewable Energy Simulator module (REnSim) supports the user in the management of the energy arising from renewable sources and the effective integration of this into the installation. It leads to maximisation in the profitability of the installation and the early detection of losses and malfunctions. REnSIM (example view in Fig. 3) provides computation for three types of renewable systems (solar thermal system: hot water, space heating and photovoltaic system).

The module requires data from the solar thermal system involving the specifications of the system, the location of the installation, the technical features of the building and the usage. It executes simulations using the following critical design parameters: historical meteorological data, building space availability data, energy use profiles and pricing data, specific renewables systems configuration technical data and equipment installation data. 


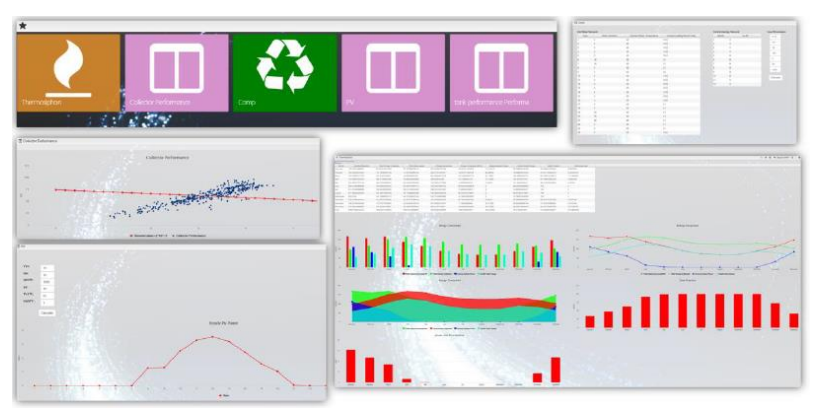

Fig. 3. REnSim graphical user interface overview.

REnSIM has been created with generic, open technologies and has no dependencies on any special use cases such as IFC files.

\subsubsection{Building Performance Simulation services}

The project platform can integrate two different building performance simulation tools, respectively named ESP-r and ModSCO. They can offer a number of performance assessment methods to evaluate differences between a pre-defined expected performance and the actual energy and comfort performance of buildings.

ModSCO (Model-Supported Control): implementation of reduced order models to assess HVAC performance of buildings by offering simulation capabilities of users/stakeholders in order to analyse and optimise HVAC control strategies in a targeted zone of a building.

Building Performance Simulator, ESP-r: identifies locations within the building where indoor environmental quality may fall below levels prescribed in international standards. It supports decision making by facility managers regarding user's complaints and potential solutions.

\subsubsection{Calibration}

The calibration module, named Calibro, allows the automatic calibration of a simulation model based on data input-output pairs for multiple simulation cases corresponding to judicious input parameter perturbation. While these input-output parameter pairs will typically depend on the intended application of the building performance simulation modules, they have no particular meaning within Calibro. The calibration method also requires measured data corresponding to performance targets (e.g. indoor conditions, energy consumption) and time-matched weather boundary conditions. Using these data, Calibro constructs an emulation (meta-model) of the connected tool and uses this to determine the input parameter values that will cause the tool to best reproduce the measured performance.

The best-fit input parameter values are then incorporated in the activated tool's input model before use in performance assessment mode. Further, the differences between parameter values before and after calibration are output as a contribution to an understanding of the causes of the performance gap. It is emphasised that Calibro is a building performance simulation tool diagnostic and is automated in its.

\subsubsection{Uncertainty and sensitivity analyses}

The module called Gap Reasoner is based on uncertainty and sensitivity analyses of building performance simulation. Thanks to him, major influences on the energy consumption are listed, which show possible reasons for an existing energy performance gap and provide energy managers an orientation to improve the building energy efficiency.

The module run simulation models with a set of uncertain input parameters and deliver statistically distributed simulation with the objective to provide a more reliable simulation result.

Making use of uncertainty analysis methods gives the user a more reliable simulation result, as input uncertainties are taken into account.

Additionally, the module provides methods for sensitivity analysis, which allows for determining the impact of varying input parameters on the output variation. More precisely, it is estimated whether, for example, the room temperature set-point or the solar gvalue of the windows is more influential for the energy consumption of a given building. The module uses this information to formulate messages to the user, which can help identifying reasons for an energy performance gap. For this also results from fault detection and diagnosis (FDD) are used to adapt the probabilities of system related reasons accordingly

\subsubsection{Display modules}

The visualisation modules are dedicated user interfaces for different groups of end users, most notably for technical users and non-technical building occupants. Several modules have their own graphical user interface, such as modules related to building performance simulation, behaviour modelling or fault detection. The purpose of these user interfaces is to provide a userfriendly configuration/presentation. The visualisation modules cover: energy management, facility management, building occupants, and Building Information Model (BIM) fields (Fig. 4).

The development of the display modules has been programmed by using different technologies and they are intended to be used on different devices. The modules for Facility Managers, Energy Managers and building occupants have been implemented with different kinds of web technology including PHP, Java, Python, Ruby as well as rich JavaScript components. The web based implementations guarantee a wide degree of flexibility and interoperability enabling an installation on diverse operating systems. Only the module BIM visualisation has been programmed in a way native to the operating system due to performance reasons. 


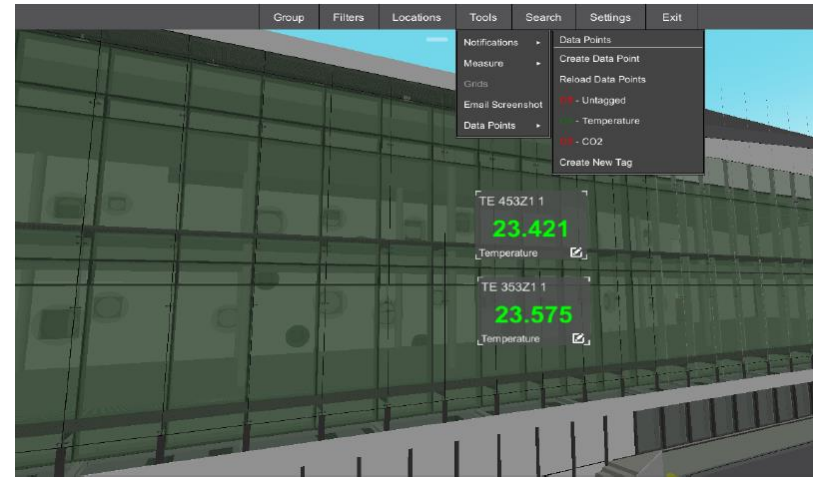

Fig. 4. View of simulation results on a 3D building image with ZUTEC module.

\section{Test and demonstration in pilot sites}

The HIT2GAP platform and related modules are under test in four selected buildings in Europe [4]. The several modules are being tested respectively in the four pilot sites, according the summary scheme in Fig. 5.

It is not possible to develop a unique BMS that can cover the needs of all buildings because the features of the buildings, by type, location and construction characteristics are diverse. The approach taken in HIT2GAP to overcome this issue is to develop an open platform that can be customized by selecting the application modules needed, or extended with new third party modules that cover new functionalities and allow integration with legacy systems in place.

To demonstrate the flexibility offered by HIT2GAP the pilot sites chosen are located in different countries with different climates and construction years. The managers of the demo sites themselves have also different expectations on how HIT2GAP could help to enhance energy efficiency in their buildings.

Each pilot site has been selected in order to have a clear and valuable strategy allowing the demonstration of one or several functionalities of the HIT2GAP platform. They are all offices buildings, our mains focus, but operating in different conditions, representative of the market.

The pilot sites have also been selected according to the fact that they are already equipped with a BMS allowing the data collection and the direct connection of the HIT2GAP platform to this BMS. The strategy is defined in agreement with the local stakeholders and in line with the existing energy management of the building.

The main characteristics of each pilot site and associated strategy regarding HIT2GAP are presented hereafter.

The Challenger Campus demonstration building is located in Saint-Quentin-en-Yvelines, in France. The climate conditions are moderate and influenced by the sea. It is a typical Western European oceanic climate, mild and wet.

The Challenger (Fig. 6) is the headquarters of Bouygues construction company. It was built in 1988 and renovated in 2014, reaching high level environmental certification levels. The site is composed by 3 main buildings ( 3 floors and 2 basements), covering an area of $65000 \mathrm{~m}^{2}$. The energy sources used by the building are ground-source, photovoltaic, thermal solar, electricity and a thermal generator (security). All buildings and the area are managed by a BMS which supervises air conditioning, lighting, ventilation, heating ventilation, sanitary water, hot sanitary water, and technical alarms (Freon gases and water leakage, technical defect, etc.)

The facility management team needed a system able to analyse the collected data over a reference period, to accurately forecast the building energy consumption and to be able to signal a malfunction before it becomes obvious. Such a tool should also enable a dynamic modification of the BMS set-up, in order to closely adapt it to all the operation condition changes: e.g. turn on or off the air-conditioning depending of the forecasted outside temperature in the coming hours.

\begin{tabular}{|c|c|c|c|c|c|}
\hline Module Name & App & $\begin{array}{l}\text { Nanogune } \\
\text { (ES) }\end{array}$ & $\begin{array}{l}\text { Challenger } \\
\text { (FR) }\end{array}$ & $\begin{array}{c}\text { Wilanów } \\
\text { Town } \\
\text { Hall }(P L)\end{array}$ & $\begin{array}{c}\text { NUIG } \\
\text { University } \\
\text { (IE) }\end{array}$ \\
\hline \multirow{2}{*}{$\begin{array}{l}\text { Fault Detection and } \\
\text { Diagnosis Modules }\end{array}$} & FDD-PCA & $\mathrm{x}$ & $\mathrm{x}$ & $x$ & $\mathrm{x}$ \\
\hline & FDD-ML & $\mathrm{x}$ & $\mathrm{x}$ & $\mathrm{x}$ & $\mathrm{x}$ \\
\hline Load Forecasting & Load Forecasting & $x$ & $\mathrm{x}$ & $\mathrm{x}$ & $x$ \\
\hline \multirow{2}{*}{$\begin{array}{l}\text { User Behaviour } \\
\text { Modules }\end{array}$} & BM-Events & & $\mathrm{x}$ & & \\
\hline & BM-ML & $\mathrm{x}$ & $\mathrm{x}$ & & \\
\hline $\begin{array}{l}\text { Energy Management/ } \\
\text { Energy Performance } \\
\text { Application }\end{array}$ & EnMS & $x$ & $x$ & $x$ & \\
\hline \multirow{2}{*}{$\begin{array}{l}\text { Building Performance } \\
\text { Simulation Modules }\end{array}$} & ModSCO & $\mathrm{x}$ & $x$ & & $x$ \\
\hline & ESP-r & & $\mathrm{x}$ & & $x$ \\
\hline Model Calibration & Calibro & $\mathrm{x}$ & $\mathrm{x}$ & & \\
\hline $\begin{array}{l}\text { Renewable Energy } \\
\text { Simulator }\end{array}$ & REnSim & & $x$ & & \\
\hline Gap Reasoner & Gap Reasoner & $\mathrm{x}$ & & & \\
\hline \multirow{4}{*}{$\begin{array}{l}\text { Visualisation } \\
\text { Modules }\end{array}$} & EM Visualisation & $\mathrm{x}$ & $x$ & $x$ & \\
\hline & FM Visualisation & $\mathrm{x}$ & $x$ & $x$ & \\
\hline & $\begin{array}{c}\text { Building } \\
\text { Occupants Module }\end{array}$ & & $x$ & & \\
\hline & BIM Visualisation & & & $\mathrm{x}$ & $\mathrm{x}$ \\
\hline
\end{tabular}

Fig. 5. Summery scheme of the modules implementation at demonstration sites. 


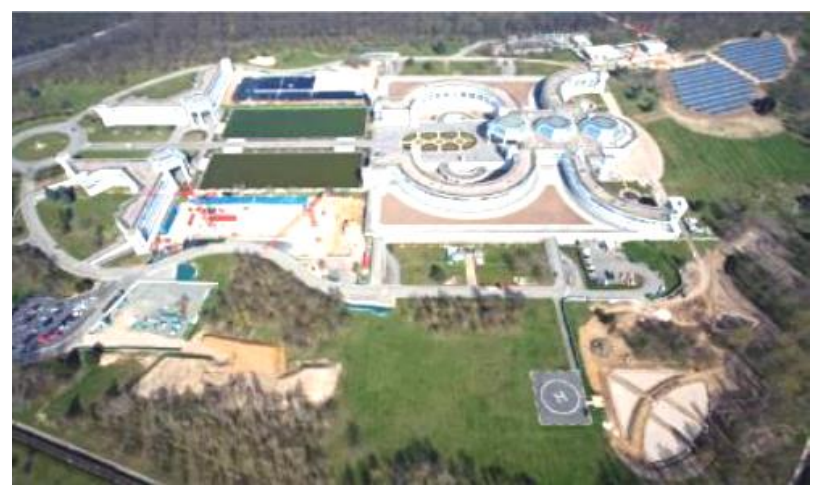

Fig. 6. View of the pilot sites Challenger Campus in France.

The NanoGUNE demonstration building is located in Donostia-San Sebastián in the north of Spain. Its climate conditions are called humid temperate climate without dry season, or Atlantic climate.

NanoGUNE building (Fig. 7) is a nanoscience cooperative research centre. The building of $7000 \mathrm{~m}^{2}$ was built in 2008. It is composed of a tower with 5 floors that contains offices and another building with 2 floors containing offices, laboratories and a clean room.

The energy sources used by the building are natural gas and electricity, and a small solar panel system is installed for domestic hot water. The HVAC, lighting system and access control are monitored. There is a BMS on site, but currently it is in process of being updated.

Thanks to the solutions proposed by HIT2GAP, it is expected to detect and reduce some cause of overconsumption. Some of the measures that are envisioned to adopt in order to reduce gas and electricity consumption are: - Limit the thermostat set-point via the BMS. Currently all thermostats are controlled by users. Change the location of thermostats and/or increase temperature sensors to have a more representative value of indoor temperature in rooms that are very big. - Use blinds in the windows to increase thermal comfort. Target user behaviour change to avoid the entrance door left open (installation of sensors can be helpful). Monitor the pumping consumption in order to know energy savings with a retrofit analyser module. - Monitor efficiently building occupancy and use it to implement energy efficient measurements such as controlling HVAC according to occupancy.

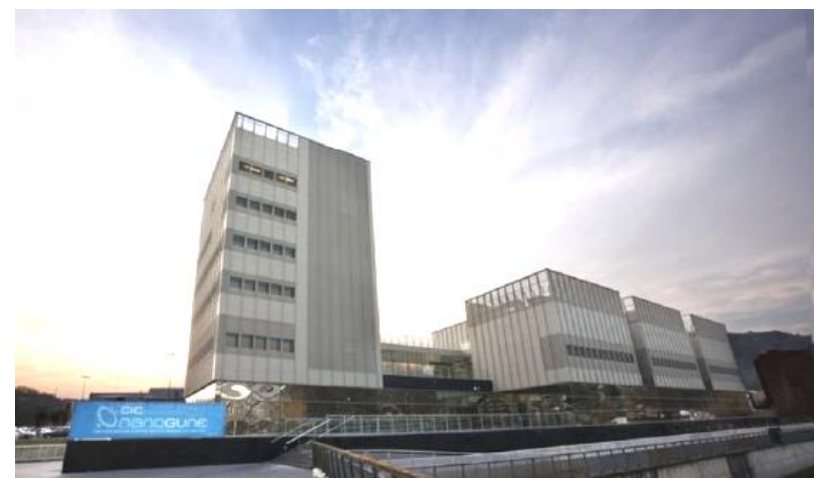

Fig. 7. View of the pilot sites NanoGUNE in Spain.

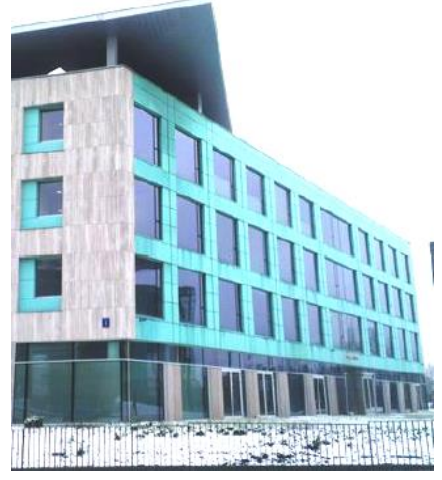

Fig. 8. View of the pilot sites City Hall Wilanów in Poland.

The demonstration building City Hall Wilanów (Fig. 8 ) is located in Warsaw in Poland. Its climate context is humid continental with cold, snowy, cloudy winters and warm, sunny, stormy summers, bordering on an oceanic climate.

The building was built in 2014. It is composed by a ground floor, 3 floors, and an underground garage. It is used as offices by on average 137 people.

The energy sources on site are district heating and electricity. The heating is powered by district heating while the cooling is electrical, with 4 chillers. Sanitary hot water is supplied by the hot water district network. The lighting system is controlled by a DALI system in the offices and it is manual in the rest of the building. There is a BMS in place that controls air conditioning, lighting, ventilation, and temperature.

Wilanów Town Hall needed to know the exact amount of water, heat and electricity used and forecasted for the next months. This functionality will help to sign more beneficial contracts with suppliers. The bills may be lower thanks to adjusting HVAC to outdoor conditions, indoor temperature and number of people occupying the rooms/offices or particular zones. The system should inform about potential saving resulting from changing HVAC settings on a display. Information about current energy use and how far the building is from optimal performance should be displayed as well. The HIT2GAP tools should help to change users' habits and extend their awareness. Moreover, the HIT2GAP solution are integrating all existing meters and give possibility to add new ones.

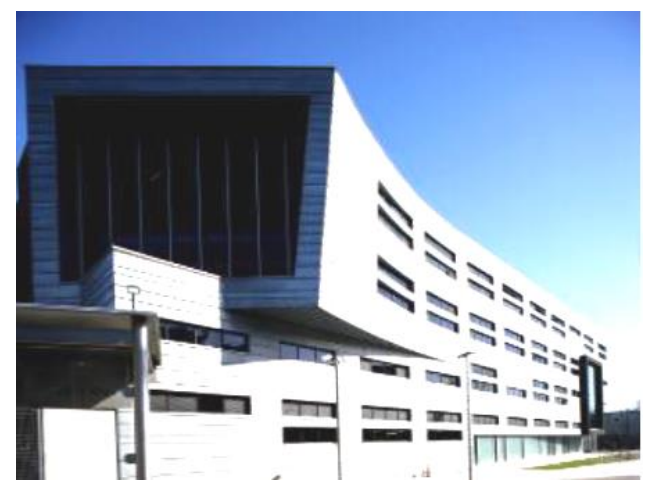

Fig. 9. View of the NUIG pilot sites in Ireland. 
The premises of the National University of Ireland (NUIG) to be piloted in HIT2GAP are located in Ireland in the city of Galway, where the climate conditions are a year-round mild, moist, temperate and changeable, due to the prevailing winds of the North Atlantic Current. It has a Maritime Temperate climate.

The building chosen is the New Engineering building of the National University of Ireland (Fig. 9), which was officially opened in 2011. It is a teaching and research facility with offices, classrooms and labs. The building has 4 floors and regarding space arrangement, it has 400 classrooms, including 3 large tiered and 25 breakout zones for group learning. It is used by 1,100 students and 110 staff members.

The energy sources on site are electricity, natural gas, biomass, district heating and solar.

Lighting is controlled automatically according to ambient luminance and occupancy and schedules. The ventilation is done through natural stack ventilation and climate wall with top-vented stack control and solar control via blinds. Currently there is a BMS in place which controls HVAC, natural ventilation and lighting. The BMS has 2086 sensors logging.

From a building energy manager's perspective, it was necessary to gauge a building's performance over time relative to an expected performance. It is the aim of the pilot site to integrate the fault detection and diagnosis, load forecasting and building simulation modules. Simulated and forecasted usage for a meter will be available via the building simulation module and forecasting module web services respectively as target data in the BMS components. The development of targets feature will evaluate the HIT2GAP data relative to the actual data; this will allow visualization of reports to show actual usage vs. target and overspend evaluation. The targets will be used to demonstrate the suitability of the computed targets (simulation or forecast) for selected meters.

For each pilot the following methodology has been followed. The consortium defined the implementation plan, after which installations and adaptations of the BMS systems took place, followed by validation of the equipment and systems. This has included activities such as: modules selection rules for the sites, selection of modules and adjustments planning for each pilot.

The process for modules selection depended on various factors and require individual selection rules. The main issue is to identify user's expectations and requirements. This selection should be done in agreement with the building owner/manager. Target user groups for each module have been defined ad the following three profiles of users: facility managers, energy managers, occupants.

Furthermore, each module has its own prerequisites. It is essential to specify types of input and output data. Finally, it is important to define if additional sensors or systems modifications are required.

After this validation tests for the core platform and for the module are ongoing in the pilot sites. Specific procedures for the validation tests have benne defined including test steps and action for each modules in order to check the systems features and functionalities. The validation results are now in the analysis phase and during the next months the detailed results can be shown.

For each pilot sites, baselines for energy consumption have been determine for the considered energy uses and energy carriers (e.g. electricity, gas, district heating). Historical measured data and values from on-going monitoring have been analysed to determine energy demand baseline related at least to two years in the period from 2015 to 2018. These values are the base for the performance gap reduction thanks to the adoption of the HIT2GAP solutions.

\section{References}

1. R. Enrich Sard et Al., Hit2Gap project, D1.9 Synthesis of the HIT2GAP methodology (2016), http://www.hit2gap.eu/documents/deliverables

2. R. Enrich Sard et Al., Hit2Gap project, D3.6 Report synthesising the description of each developed module (2017), http://www.hit2gap.eu/documents/deliverables

3. International Organization for Standardization, SO 50001 - Energy management (2018)

4. B. Rudzki, J Żach, et Al., Hit2Gap project, D5.2 HIT2GAP prototype implementation report (2018) 